\title{
TreasAR Hunt - Location-Based Treasure Hunting Application in Augmented Reality for Mobile Devices
}

\author{
Adina-Maria Oros \\ Technical University of Cluj- \\ Napoca \\ Str. G. Barițiu 28, 400027, \\ Cluj-Napoca, România \\ adinaoros98@gmail.com
}

\author{
Adrian Sabou \\ Technical University of Cluj- \\ Napoca \\ Str. G. Barițiu 28, 400027 \\ Cluj-Napoca, România \\ adrian.saboules.utcluj.ro
}

\begin{abstract}
TreasAR Hunt is a mobile application that blends together Augmented Reality and location tracking methods. It addresses to both technical and non-technical users. The application comes to enhance the learning process by creating an immersive environment that keeps the user interested and engaged. Thanks to the type of game it models, a treasure hunt, and to the fact that it involves interaction between the user and its surroundings, the application can also be used in tourism, as a city guide. In addition to that, it can be seen as a team-building tool, that dares players to collaborate. This paper describes the project's objectives, its use cases, core functionalities and development phases. User testing results are provided and interpreted at the end, so to conclude how does the application fit in the stated scopes.
\end{abstract}

\section{Author Keywords}

Augmented Reality; Marker-based detection; Location Based Services; Location tracking; Unity 3D; Educational application; Training application;

\section{ACM Classification Keywords}

H.5.m. Information interfaces and presentation: HumanComputer Interaction; Interaction Techniques;

\section{General Terms}

Augmented Reality; Location Based Services; Image Tracking;

DOI: 10.37789/rochi.2021.1.1.9

\section{INTRODUCTION}

One cannot talk about trends in technology without saying Augmented Reality (AR). In addition to that, AR ready devices are continually becoming better and more accessible. The technology just finds its way into the industry - from gaming to healthcare, from entertainment to education and so many more. Thanks to the continually improving computer software and hardware, AR has developed very fast and the research started going beyond the theoretical stage, into the mass, industry production. It is here to stay and transform people's way of interacting with the surrounding world.
A lot of AR's power comes from the fact that it is highly visual and highly reactive. As humans, we acquire the most information through our vision, while our attention is drawn by things we are able to interact with. Intelligent interaction, real-time tracking, 3D models and sensing are just a few of the technical means AR technology is using. The physical world is combined with computer generated virtual information like 3D models, videos, images, music etc. into an interactive environment.

Treasure Hunt is a game that can be played in groups, or just by individuals. Its scope is to get players familiar with a subject or area. During the game, players must search for certain objects while following given hints. The objects are usually chosen so to make things more interesting and complicated - unusual, hard to notice objects like a bar menu, a door sticker, a detail from the upper corner of a room, it can be literally anything. These can be scanned by device's camera and enhanced through real time virtual images, animations, music and so on. The hints remain the same, but all the surroundings can benefit from the power of AR. The game is played over a certain area, which gives the possibility to make it into a location-based application.

Location based services (LBS) make their way into the technological world around the same time as Augmented Reality (ActiveBadge[1]). Many changes and improvements in the technology have been made public since and as communication technology continues to evolve, more advances in the field are prone to appear, as more and more studies are conducted in this direction. Just as for AR, Location Based Services have been looked at from different perspectives and used in many industries: from transport, to education [2]. Integrating LBS in the application helps in creating a game that surrounds you.

Since the Internet took over and information became accessible for everyone at any moment, it is getting harder to get students involved. Traditional learning methods are tedious and not so interactive. Most studies now favor AR training and learning techniques in comparison with traditional learning methods [3], because it helps one acquire skills in problem solving and creativity more easily, offering a different learning experience. It can increase on content understanding and memory preservation, as by nature, it's 
harder to forget rich visuals. Big steps have been already taken in this direction, accelerated by the 2020's pandemic, when the educational institutions had to find ways of keeping students engaged while being in their own home and under no professional supervision.

The project comes in the purpose of helping people learn while exploring and explore while they learn. The game involves interaction between the user and its surroundings, such that the application can be very easily used in tourism, as a city guide and in training, as a team-building game. The application addresses to both technical and non-technical users, such as students, tourists or people that work in a company.

\section{RELATED WORKS}

Augmented Reality is known to have enhanced effectiveness and attractiveness of teaching by combining the virtual and physical worlds. Due to its highly visual character, AR comes to help teachers explain a subject by displaying realtime information that is easier to understand and harder to forget. There is an AR app for almost any subject, including biology, zoology, chemistry, physics and so on. A good example of such an application is described in "Application of Augmented Reality Technology in Biological Learning" [4]. The described application displays the $3 \mathrm{D}$ version of the objects contained in the biology book (Figure 1).

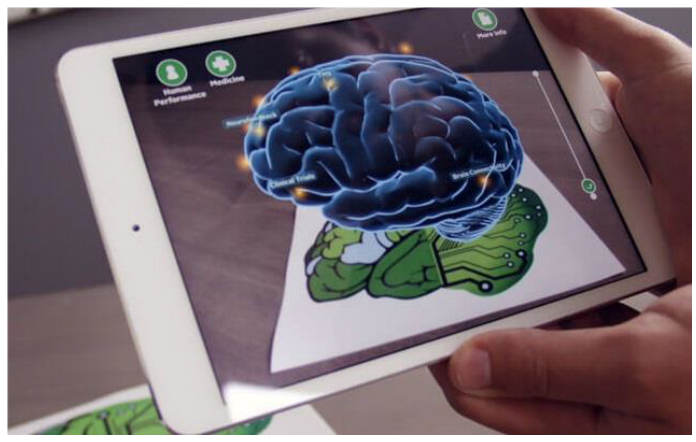

Figure 1. 3D brain displayed over its 2d representation [4]

In the attempt to move digital games outside, into the real world, game designers started to integrate location providers into their games. Pokémon GO is a free-to-play game launched by Niantic in July 2016. It is an Augmented Reality mobile application that uses Location Based Services. The scope of the game is to capture virtual characters that can be found in real locations, such as stores, parks or streets. The application is using GPS capabilities in order to display information about player's geographic location on the map. When examining motivations to play Pokémon GO, under several benefits, physical activity is cited most often. It is considered that the game promotes an active lifestyle, being seen as a potential helper in fighting obesity and inactivity [5].

HUNT is an example of AR treasure hunt application. It is the result of a study that concentrates on exploring learning opportunities through an activity based on orientation. The application is designed to work only for Iphone devices, but is addressed to both creators and players of the game. It consists of a web admin interface, that offers possibility to create scavenger hunt games or update existing ones, directly uploading them into the mobile HUNT application. Through the mobile application, users are able to play the game. It provides augmented content for each target found and computes the scores. The targets are given in a list at the beginning of the game, each object being assigned different values. After a successful find, the player is directed to the next target and so on, following the general principles of a treasure hunt. When the game is finished, a summary page is displayed. Hunt application is here to show how Augmented Reality technology can be used to add value as an educational component [6].

\section{CONCEPTS}

Treasure hunt, often called Scavenger Hunt, is designed to get players explore a certain area, or to get them familiar with a topic of interest. Hidden objects need to be found by following some hints. Each object reveals the hint to the next one and so on. The game is finished when all the treasures are discovered.

Augmented Reality (AR) is an emerging technology that combines elements from the real world with computer generated perceptual information, including visual, auditory, somatosensory, haptic and olfactory.

Computer Vision is an interdisciplinary scientific field that seeks to understand and automate tasks that human vision system can do.

Tracking methods represent ways of locating, recognizing and interacting with points of interest from an environment. Accuracy of applications that use AR technology is strongly dependent on the quality of the implemented tracking methods.

Non-photorealistic rendering is a visualization technique that does not aim for realism. It uses clear borders and bright colors, catching human attention immediately. Nonphotorealistic rendering gives good results in situations where the augmentation is used in order to provide instructions. With the use of this technique, the user can concentrate on the information to be conveyed [7].

Location Based Services (LBS) offer information about device's location. These details are usually transferred via Global Positioning System (GPS)

Architectural patterns are reusable solutions to commonly occurring problems in software engineering.

Singleton objects are restricted to only one single instance. 


\section{THEORETICAL FOUNDATION}

Marker-based tracking methods

Marker-based tracking methods rely on recognizing shapes, images, 2D visual representations that are usually static. Markers are brought to life in the AR context with the help of computer vision. The 3D objects that overlay $2 \mathrm{D}$ markers can be moved around, played with or even used as buttons that trigger actions. The advantages regarding this type of tracking are its stability and ease of implementation. If prepared correctly, the markers offer stable tracking and qualitative AR experiences. The trigger image must be unique, thus restricting the marker to a single object. This method is sensitive to environment changes [8], making the outdoor image recognition more complicated. For robust applications, the type of markers that are often used are black and white, have thick borders and are unique by pattern (Figure 2).

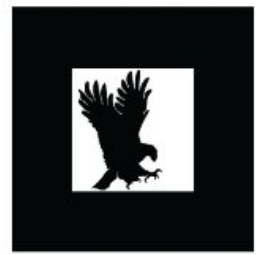

(a) Template Marker

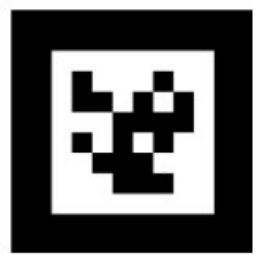

(b) Bar-code Marker

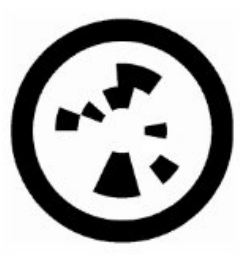

(c) Circular Marke
Figure 2. The three types of black and white markers [9]

Location-based gaming

Location Based Gaming (LBG) maps game's virtual environment into the real world. Application's contents adapt to player's location, which is updated in real-time via GPS [2].

\section{Client-Server}

Client-Server system is described as an architecture that contains both server and clients. Each of them perform different functionalities, but exchange information, creating an inter-process communication [10]. More specifically:

- Client-side is running on the end-user device. It contains the User Interface (UI), which defines how the user interacts with the application. It might use some of device's resources like internal storage.

- Server-Side receives and handles requests from the clients. It contains the logic needed in order to send the right response in terms of data. It doesn't have a UI, but rather an Application Programming Interface (API). Moreover, the server contains a database in which to store all application related data.

Model-View-Controller

Model-View-Controller (MVC) is an architectural pattern used to separate internal representation of data from the way this information is presented to and accepted by user. The system gets divided into three main components:
- Model - contains all data related models that might be used by a user;

- View - contains all the UI elements the user can interact with;

- Controller - defines an interface between the model and view components. It processes business logic and handles requests. It manipulates the data using Model and interacts with the View component to render output.

\section{PROPOSED SOLUTION}

Objectives

Project's major objective is to combine Augmented Reality technology with Location Based Services in the development process of an interactive treasure hunt application. The result aims to get users into the immersive experience of an augmented environment, while keeping them connected to the physical world. Connection is kept by continuously providing their spatial location. The application should be portable and easy to use; it should offer an alternative to the common way of playing a treasure hunt, while enhancing parts of the learning process through exploration.

\section{Use Cases}

Three principle use cases have been identified (Figure 4):

- Registration \& Authentication;

- Reveal target location;

- Recognize target and collect treasure.

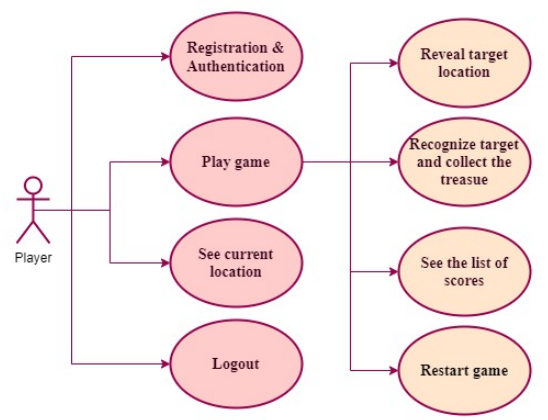

Figure 3. TreasAR Hunt use-case diagram

\section{Development Tools}

\section{Unity Engine}

Unity is a cross platform game engine that drives the democratization of game development [11], by making available many complex documented tools. It combines user interface with architectural and object-oriented designs to control and visualize video games; it also contains an IDE component supporting $\mathrm{CH}$ scripts. Base properties to note [12]:

- Unity is compatible with over 30 other platforms, like: iOS, Android, Windows, Playstation etc.;

- It has all the tools to create a video game: asset store, IDE, 3D modeling editor, texture editor;

- It supports both virtual and augmented reality; 
- It offers the possibility to create the User Interface within the platform, using 2D sprites and a drag and drop kind of editor;

- Software Development Kits (SDK) can be integrated to bring more capabilities to the platform.

Vuforia SDK

Vuforia Engine is an Augmented Reality Software Development Kit (SDK) for mobile development. This package offers computer vision that helps in recognizing objects and images, but also interact with the world space. Some notable capabilities:

- Real-time tracking makes the interaction between the virtual objects and real world possible;

- It supports both 2D and 3D target types, marker-based and markerless;

- It is able to reconfigure targets at runtime;

- Includes web-based Developer Portal, where image targets can be uploaded and rated in terms of detectability.

\section{Firebase Realtime Database}

Firebase is a Google-backed application development software. Firebase's Realtime Database is a NoSQL database that provides us with the possibility to synchronize data between active users in real-time. JSON format is used in order to store data. The database is cloud hosted and users receive updates of data continuously.

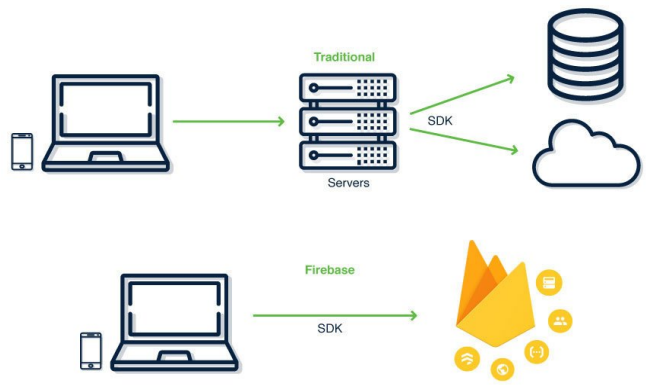

Figure 4. Traditional vs. Firebase database [13]

\section{Adobe Photoshop}

Adobe Photoshop is a raster graphics editor that offers a variety of tools with multiple image-editing functions. Its Shape tool is the one used for creating the sprites integrated in the design of our User Interface.

\section{Design and Implementation}

\section{Conceptual Architecture}

This application is dynamic in terms of data and user events, which means that it must respond to player's actions in realtime. In order to achieve this kind of interaction, we combine the model-view-controller type of architecture with the client-server approach of retrieving data (Figure 6).

The application that requests data from the server is the client. It is created within Unity's 3D platform. In order to use Firebase's Realtime Database, its SDK has to be integrated within the Unity project. Authentication, save and retrieval of data are all assured by Firebase, while programmers have to assure that the right request is made at the right time.

The application makes use of MVC [14] pattern to isolate business logic from the user interface. These two parts of the system communicate with the help of controllers, that in this case, are implemented under the form of C\# scripts.

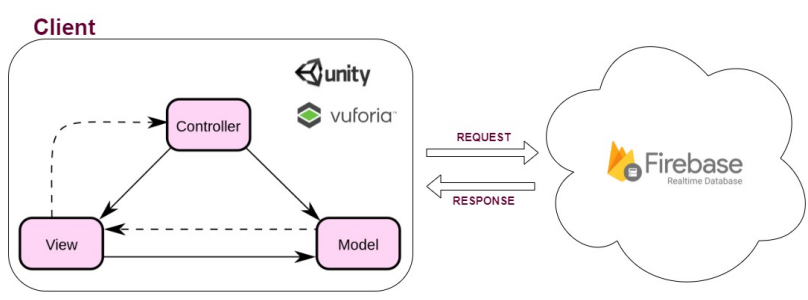

Figure 5. Application's MVC architecture using Firebase server

Augmented Reality

The final application uses Augmented Reality in order to visually enhance the perception of real world. Translating treasure hunt actions to an AR type of game, the players should be looking for virtual objects that are found in real places. Once the user gets to the correct place, the application augments the reality and displays the hidden treasure as a virtual object.

Vuforia SDK is used in order to recognize target images and overlay 3D objects. The cloud-based database of targets is accessed through web services and the device-based target database is downloaded and integrated directly with the application (Figure 6).

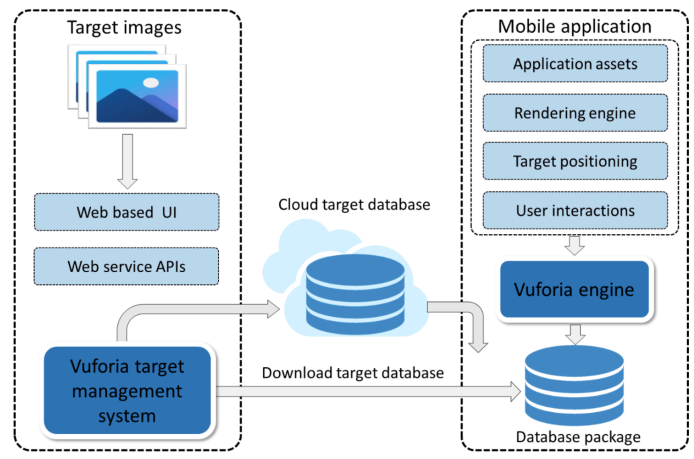

Figure 6. Vuforia SDK architecture [3]

Vuforia SDK's data stream is composed by four modules inputting, database, matching and tracking, output render. The image captured by the mobile device must match an object from the database, so to render the virtual objects. Images are recognized based on their natural features (Figure 8). 


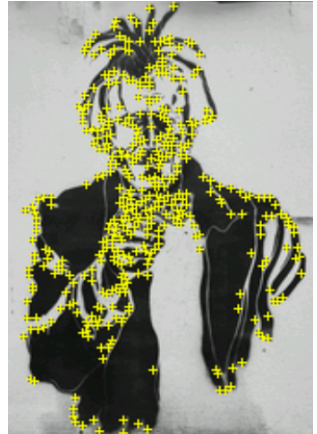

Figure 7. Detected features of Vuforia target

In order to score a high detectable rate, images need to have sufficient detail. Once an image gets uploaded into the database, it is evaluated by the Target Manager - the image from Figure 8 scored 5/5 stars.

Our image targets are represented by objects like building plates, street art, books, statues. The images were preprocessed so to not contain changing background information and when needed, contrast was adjusted.

\section{Location tracking}

Location based services are used in order to extend player's experience into the real world. The application displays device's current location at each moment in time. In order to do that, Unity's LocationService class is used. The bond between the application and device's GPS is done through a C\# script from the Unity project. If access to the location is permitted (if not, the application should ask for it), at Start() call, location details are obtained by calling functions of LocationService class.

\section{Main Functionalities}

Authentication is composed of two modules: Login and Register. In order to play the game, a user needs to log into the application by introducing their account credentials. In case the user does not have an active account, they can register. The application this paper aims to describe is mostly dedicated to people that have low to none knowledge in technology. The system doesn't hold any important user data, the scope of accounts being to keep user's progress in the game; thus it was considered that a nickname, email and password is enough information to register as a treasure gatherer. Authentication is done with the help of FirebaseAuthentication, which is making secure authentication easy to build in.

Login and register ask for logout functionality. After user got logged into the application, they should be able to logout from it easily and at any time. Logout is made available as an option of the contextual menu that's found within the game.

Reveal target location. Treasure Hunt is a game based on searching. Each treasure has its location specified. If users cannot find the target, its location can be revealed, but only after providing answer to a question directly subjected to the theme of the game (Figure 9). If answered correctly, the location is revealed without any loss. If the answer is incorrect, player loses some score points.

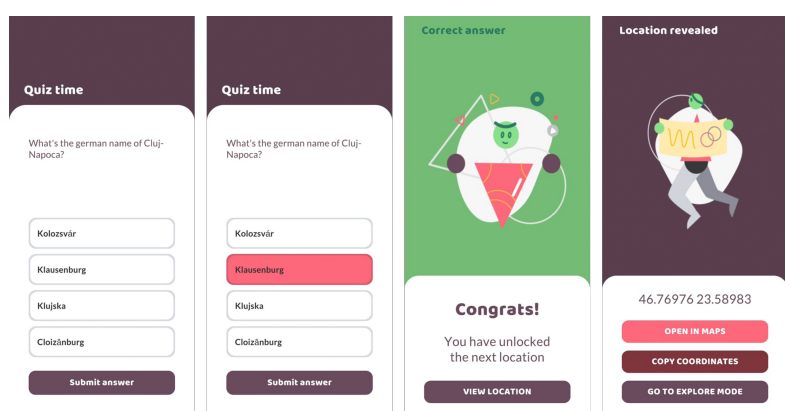

Figure 8. Example of a correctly answered quiz

In order to provide a smooth navigation, when location gets revealed, it can be opened, directly from the game, in a navigation application that's been already installed (e.g. Google Maps).

The quiz is stored in the database as a child of the target it is bonded to. In order to be complete, it contains one question, four answer options and one correct answer. It is retrieved from the database with the use of a Quiz class that's been created.

Collect treasure. Each treasure has an image assigned. In order to add an image target, Image is selected from Vuforia Engine options. In the inspector, its size is changed so to match the real size; one unit of size in Unity is one meter in reality. Each target is of type predefined and can be found in the database that has been previously imported from Vuforia Development Platform. After the image target is set, the objects that will be overlaid to it are added as children: three information bullets and the award. Each information bullet will trigger the display of information.

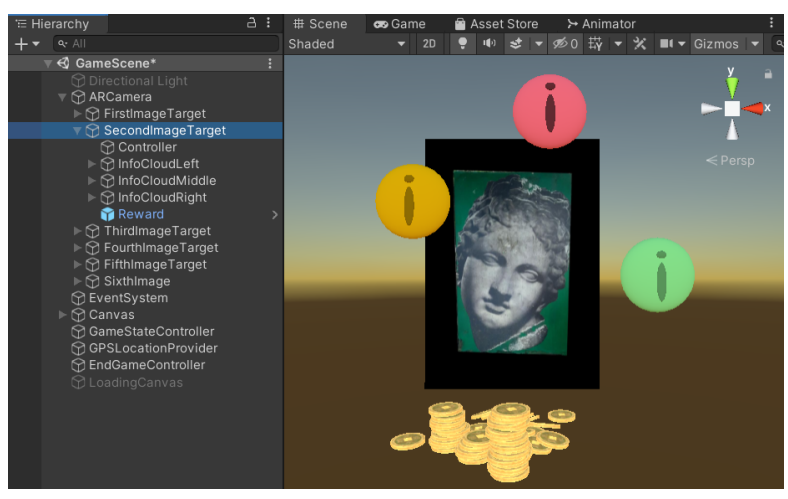

Figure 9. Image target as seen in the Unity 3D scene editor, together with the hierarchical representation of its children

Score listing. When the game is finished, player's final score is computed and added to the existing list of scores. Because the list of scores changes each time a new player finishes the game, a dynamic approach is used, where the position of 
each entry is placed at a location calculated based on its position in the array. This is controlled through a C\# script.

Real-time location display. TrasAR Hunt is displaying device's location, in terms of coordinates, in real-time. In order to make the bond between device's GPS an the Unity application through LocationService class of Unity Engine, a C\\# script is used. It is important to note that location permission is needed and this script holds the logic that asks for it.

\section{Controllers}

Within the application, there are two very important controllers: one manages the game's state and one the scene.

GameStateController: manages the information that is rendered on the gameplay screen. It keeps track of score, current hint and target location quiz. The script is assigned to the $3 \mathrm{D}$ controller object that's found in the main Game Scene. The controller holds instances of both LocalService and GameService singletons. The LocalService is instantiated so the Logout functionality can be accessed by user from within the game. The GameService class is used to offer information such as player's score, level and hint. It also contains the logic needed to Restart game and update user's information in the database, while the output rendered is changed to the start game screen.

GameSceneController: manages the UI components and game objects that appear at the moment a treasure is found. Each image target has its own game scene controller. When the target image is recognized, three information bullets appear. When one informative text is read, the treasure is displayed. The user can continue on accessing the information bullets only if it didn't collect the treasure. When the award gets collected, the controller destroys the current target and activates the next one.

\section{USER INTERFACE}

Because User Interface is a central issue regarding the usability of a software product [15], we concentrated on creating an intuitive user experience. It has to offer:

- Context-users should be aware of the game's scope and how to play it;

- Clarity - users with little to none knowledge in technology should be able to play the game and access all its functionalities;

- Easy navigation - users should easily go through the application and should always be aware of its state.

The interface design is consistent, it uses the same color palette, fonts and UI element styles throughout the whole application.

Creating User Interface based on sprites

A sprite is a 2D image that can be attached to an element. By default, these elements are not styled as we would want them.
All the UI elements within the final application have been created with the use of sprites, so to match the created design.

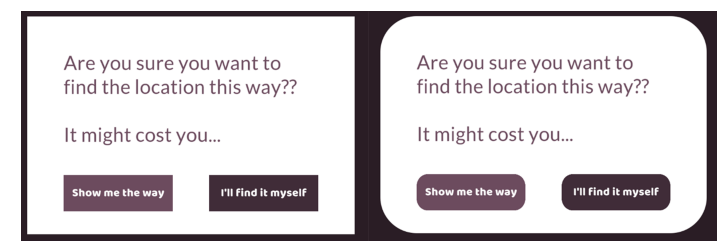

Figure 10. Application's Confirm location reveal dialog before and after using customized sprites

\section{Screen during game}

During the game, the frames captured by device's camera are displayed in real-time. Few UI elements are used so to: display the score and three icons that offer accessibility to application's functionalities: view hint, reveal target location and a menu with three options (logout, restart the game, exit).

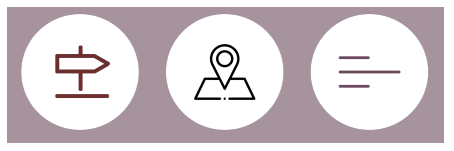

Figure 11. Hint, Location Reveal and Menu icons

The user should be able to keep track of its progress in real time, so the score is always displayed on the upper right corner.

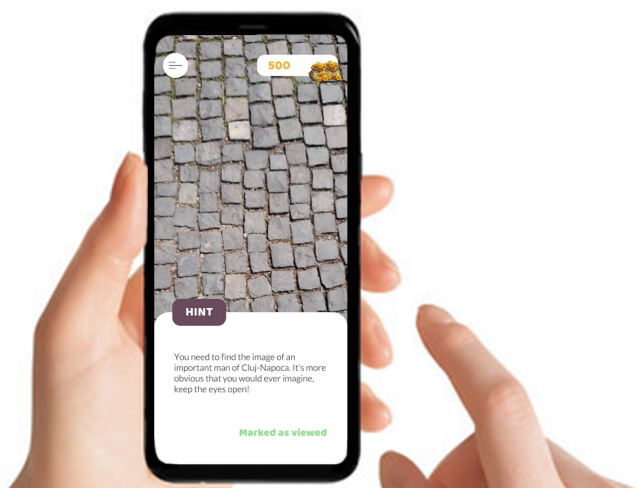

Figure 12. Hint displayed on the application's screen during game play

\section{Resources needed}

TreasAR Hunt game can be played on smart mobile devices that support Augmented Reality capabilities. Because the application is using a cloud-based storage, during the game, devices must have the internet connection enabled and running. Because the game is based on player's location, the user must find itself within the specified game area. 


\section{USER TESTING}

The application was given all the data needed in order to become a city guide of Cluj-Napoca, Romania. Five image targets were chosen from within the city and each of them got documented with the necessary amount of information.

The group of testing users was composed of five students, each of them from a different field of study: psychology, pharmacy, medicine, computer-science and robotics. They are all part of the Romanian scouts community and have noticeable experience in organizing activities and exploration.

Before the testing started, they downloaded the apk file containing the application were told the scope of it. Everybody seemed very excited and appealed by the application's idea. At the beginning, each user took a bit of time to get into the game play and see what each UI element is for. By the time they found the first treasure, it became pretty clear how the game works. At the second station, a user found and used the reveal target location functionality and used Google Maps to get to the treasure.

Beginning with third target, testing players began to feel familiar with the game play and its functionalities; they became more focused and better oriented, while enjoying the game. It was observed that after trying each functionality, users began to switch methods of finding the treasure, becoming more efficient. For example, in situations when, after reading the hint, there was not a clear idea in which direction to head to, the main choice was to answer the quiz, taking the risk of losing points.

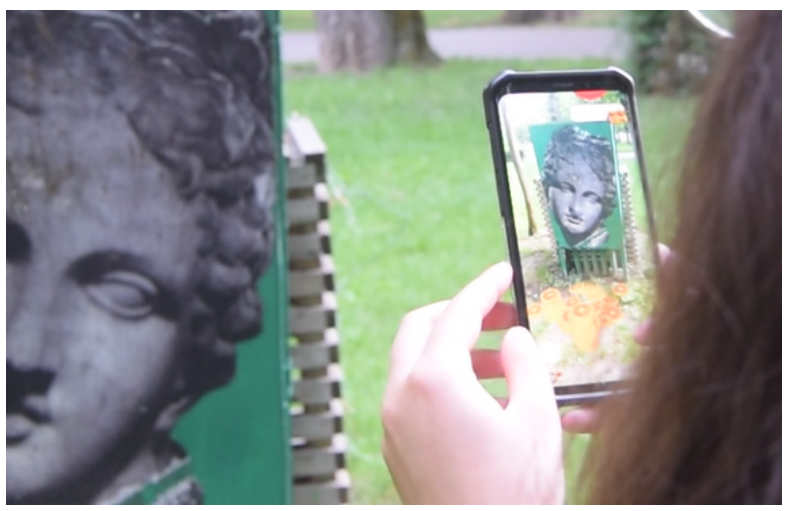

Figure 13. Photo taken during TreasAR Hunt user testing

After the game has finished and the last treasure got collected, testers were asked to evaluate the experience, based on a questionnaire we provided.

They were asked questions like:

- What's something you liked about the game?

- How would you improve it?

- Could you find another use case for this application?

- Considering another situation, would you play the game again?
- Would you recommend it to a friend?

They were asked to evaluate, on a scale from 1 to 5 :

- $\quad$ how much did they enjoy playing TreasAR Hunt;

- how much did they like the User Interface design;

- how easy did they manage to go through the application;

- how suitable did they think the application could be in the scope of learning;

- how suitable did they think the application could be in the scope of a city guide;

- how suitable did they think the application could be in the scope of a team building tool;

- $\quad$ how did they find the Augmented Reality experience;

- how did they feel about the location oriented approach.

\section{Results}

All testing users enjoyed playing the game and each of them would recommend it to a friend. One user stated that even if they've been living in Cluj-Napoca for some years now, the experience helped in finding out more about the city. The psychology students, who also teaches kids in school, said that they would use the application in the learning process.

In terms of use cases, the majority said that the stated ones are very applicable, without mentioning anything else. Except for one member of the testing group, that said the application could be used in the scope of a Pub Crawl.

The group seemed to agree on most parts, by evaluating with a 5 the AR experience, the location based approach and application's applicability as a city guide and as a team building tool.

In terms of improvements, a user said they would add more target locations, confirming again that the game was fun to play. Yet another user stated that the application could have the AR models improved and would make some minor design changes., without specifying them.

By analyzing all results, it was concluded that there could be improvements made in terms of game navigation and that some User Interface component can become better. Mostly, in terms of its stated scope, testing users agreed that the product addresses the needs.

Overall, users' experience was a pleasant one, they considered the application useful and were excited to be the first who tried it.

\section{CONCLUSION}

Augmented Reality is an emerging technology. With its help, activities are becoming much more motivational and effective. Overlaying virtual elements into the real world transforms learning methods into attractive activities. In general, AR-based applications are known to be appealing for human attention due to their high level of interactivity. By pairing AR methods with Location Based Services, user's experience is extended out into the real world even more. These two technologies are a key to construct attractive and effective methods to explore the surroundings, while learning new things. 
TreasAR Hunt is an application for Android devices, which models a scavenger hunt type of game. It came in the need to offer a tool that can enhance the learning process, transforming traditional methods into fun and attractive activities to which students become more involved. More than that, the application can be easily transformed into a virtual cityguiding activity, or be used in team-buildings.

The two emerging technologies, Augmented Reality and Location Based Services, were blended into a standalone mobile application. The game has a User Interface that helps in guiding players through the game. UI elements are combined in order to construct full screens that offer context, help to authenticate into and navigate through the application.

On the whole, the main objectives were accomplished. The group of testers evaluated TreasAR Hunt as being useful fun, interesting and a good fit for the stated scopes.

\section{ACKNOWLEDGMENTS}

We thank all volunteer testers and all publications that made their papers available for our use.

\section{REFERENCES}

1. Want, Roy \& Hopper, Andy \& Falcao, Veronica \& Gibbons, Jonathan. (1992). The Active Badge Location System. ACM Trans. Inf. Syst.. 10. 91-102. 10.1145/128756.128759.

2. H. Huang, G. Gartner, J. M. Krisp, M. Raubal, and N. V. de Weghe, "Location based services: ongoing evolution and research agenda", Journal of Location Based Services, no. 1748-9725, pp. 63-69, 2018. [Online]. Available:

https://doi.org/10.1080/17489725.2018.1508763.

3. Afnan; Muhammad, K.; Khan, N.; Lee, M.-Y.; Imran, A.S.; Sajjad, M. School of the Future: A Comprehensive Study on the Effectiveness of Augmented Reality as a Tool for Primary School Children's Education. Appl. Sci. 2021, 11, 5277. https://doi.org/10.3390/app11115277.

4. Arslan, Reyhane \& Kofoğlu, Muhammed \& Dargut, C. (2020). Development of Augmented Reality Application for Biology Education. 62-72. 10.36681/tused.2020.13.

5. Marquet O, Alberico C, Adlakha D, Hipp JA. Examining Motivations to Play Pokémon GO and Their Influence on Perceived Outcomes and Physical Activity. JMIR Serious
Games. 2017;5(4):e21. Published 2017 Oct 24. doi:10.2196/games.8048.

6. Y. Lu, J. Chao and K. Parker, "Hunt: Scavenger hunt with augmented reality", Interdisciplinary Journal of Information, Knowledge, and Management, vol. 10, pp. $2135,012015$.

7. S. Siltanen, "Theory and applications of marker-based augmented reality", VTT, Technical Research Centre of Finland, 2012. [Online]. Available: https://cris.vtt.fi/en/publications/theory-andapplications-of-marker-based-augmented-reality-licenti

8. S.-W. Jang, J. Ko, J. Lee and Y. S. Kim, "A Study on Tracking and Augmentation in Mobile AR for e-leisure", Mobile Information Systems, vol. 2018, no. 4265352, p. 11, 2018.

9. Le, Robert \& Nguyen, Minh \& Tran, Huy \& Yeap, WaiKiang. (2017). Pictorial AR Tag with Hidden MultiLevel Bar-Code and Its Potential Applications. Multimodal Technologies and Interaction. 1. 20. 10.3390/mti1030020.

10. Sulyman, Shakirat. (2014). Client-Server Model. IOSR Journal of Computer Engineering. 16. 57-71. 10.9790/0661-16195771.

11.M. Sheridan, "Unity Drives the Democratization of Development in 2016 With Eight Unite Conferences Globally", https://unity.com /our company/newsroom/unity-drives-democratization development-2016-eight-unite-conferences globally,[Online; accessed 26-June-2021]

12."unity | documentation”, Unity Documentation, 2020. [Online]. Available: https://docs.unity3d.com/Manual.

13. "What is Firebase and How Does it Work?" https://www.hammermarketing.com/what-is-firebaseand-how-does-it-work/, 2021, [Online; accesses 27-June2021].

14. Ram Naresh Thakur and U S Pandey, "The Role of Model-View-Controller in Object Oriented Software Development", Nepal Journal of Multidisciplinary Research, June 2019

15.R. Oppermann, User-interface Design. Berlin, Heidelberg: Springer Berlin Heidelberg, 2002, pp. 233248. [Online]. Available: https://doi.org/10.1007/978-3662-07682-8 15 